in vivo $36: 723-730(2022)$

doi:10.21873/invivo. 12758

\title{
Significance of HER2 and VEGFR2 in Early-stage Endometrial Cancer
}

\author{
HYO JUNG AN $\mathrm{N}^{1,2,3^{*}}$, DAE HYUN SONG ${ }^{1,2,3^{*}}$, YU-MIN KIM ${ }^{3}$, \\ HYEN CHUL JO ${ }^{4,5}$, JONG CHUL BAEK ${ }^{3,4,5}$ and JI EUN PARK ${ }^{3,4,5}$ \\ ${ }^{1}$ Department of Pathology, Gyeongsang National University Changwon Hospital, Changwon, Republic of Korea; \\ ${ }^{2}$ Department of Pathology, Gyeongsang National University School of Medicine, Jinju, Republic of Korea; \\ ${ }^{3}$ Institute of Health Science, Gyeongsang National University, Jinju, Republic of Korea; \\ ${ }^{4}$ Department of Obstetrics and Gynecology, \\ Gyeongsang National University School of Medicine, Jinju, Republic of Korea; \\ ${ }^{5}$ Department of Obstetrics and Gynecology, \\ Gyeongsang National University Changwon Hospital, Changwon, Republic of Korea
}

\begin{abstract}
Background/Aim: The majority of patients with early-stage endometrial cancer (EC) have a good prognosis, but recurrence does occur despite diagnosis at an early stage. There is a growing need for early diagnosis of EC and novel treatment options. Materials and Methods: Human epidermal growth factor receptor 2 (HER2) and vascular endothelial growth factor receptor 2 (VEGFR) expression in microarrays of patient EC tissue was examined in association with clinicopathological data. HER2 and VEGFR2 expression in Ishikawa cells and differences in migration and proliferation of cells with HER2-knockdown were evaluated. Results: Higher expression of VEGFR2 was associated with lower International Federation of Obstetrics and Gynecology grades $(p=0.044)$. The positive correlation between HER2 and VEFR2 expression was statistically significant in T1 stage $(p=0.002)$ and International Federation of Obstetrics and Gynecology grade 1 tumors $(p=0.004)$. Wound-healing assays revealed that HER2 loss in Ishikawa cells reduced confluence compared to that of control cells. Conclusion:
\end{abstract}

This article is freely accessible online.

*These Authors contributed equally to this work.

Correspondence to: Ji Eun Park, Ph.D., Department of Obstetrics and Gynecology, College of Medicine, Gyeongsang National University, Gyeongsang National University Changwon Hospital, 11, Samjeongja-ro, Seongsan-gu, Changwon-si, Gyeongsangnamdo, Republic of Korea. Tel: +82 552143783, Fax: +82 552143253, e-mail: obgy@gnu.ac.kr

Key Words: Endometrial cancer, immunohistochemistry, VEGFR2, HER2, biomarker.
The association of VEGFR2 and HER2 expression in early EC was elucidated. This study shows that the measurement of VEGFR2 expression may be useful in the preoperative assessment of EC and suggests the possibility of anti-HER2 therapy for $E C$.

Endometrial cancer is the sixth most common cancer in women and the most common malignancy of the female genital tract worldwide (1). According to the National Cancer Registry (2), the incidence of endometrial cancer in Korea continuously increased each year from 1999 to 2009 and particularly from 2014 to 2017; moreover, the incidence of endometrial cancer in young women has been rapidly increasing. Because symptoms such as abnormal uterine bleeding appear at a relatively early stage, many newly diagnosed patients have a good prognosis, with a 5-year survival rate of $80 \%$ or higher due to early tumor stage at diagnosis (3). However, $20 \%$ of endometrial cancers recur, and $6-11 \%$ of patients who experience recurrence are diagnosed in early-stage, low-risk groups (4). The median survival of women with recurrent or metastatic endometrial cancer is only approximately 12 months, and the very limited number of approved anticancer drugs contributes to the poor prognosis (5). To date, the most commonly used drugs for chemotherapy are platinum compounds, taxanes, and anthracycline (6).

The majority of tumors classified as type I endometrial cancer (approximately $80 \%$ to $90 \%$ ) develop in the background of unopposed estrogen stimulation due to obesity or an anovulatory cycle. Type I tumors are often preceded by endometrial hyperplasia, most commonly show low-grade (grade 1 or 2) endometrioid morphology and are estrogen receptor- and progesterone receptor-positive. Type II tumors, which are characterized by a high histological 
grade, usually occur in older patients on a background of endometrial atrophy and are associated with estrogen independence, estrogen receptor- and progesterone receptornegative status, and a worse prognosis.

One of the most important mechanisms in tumor growth and metastasis is angiogenesis. Therefore, there have been numerous attempts to necrotize cancer cells using drugs that inhibit vascular proliferation (7-10). The most representative drug is bevacizumab, a humanized monoclonal antibody that targets vascular endothelial growth factor (VEGF); this US Food and Drug Administration-approved drug has been used to treat advanced colorectal carcinoma since 2004 (11-14). In addition, ramucirumab targets VEGF receptor 2 (VEGRF2) and was recently approved by the US Food and Drug Administration for clinical use in various solid cancers, including gastric, lung, and colorectal. For endometrial cancer, measurements of VEGF levels, either in the tumor or plasma, are used to assess cancer stage and prognosis, and studies are ongoing to determine the mechanism of abnormal VEGF expression (15-17).

Amplification of the HER2 gene is a major mechanism of HER2 protein overexpression. HER2-positive breast cancer cells have a high proliferative index, histological grade, and nuclear grade. Trastuzumab is a drug that targets HER2, especially in breast cancer and stomach cancer (18). When patients with HER2-positive cancer are treated with trastuzumab, most experience dramatic tumor regression (19). Therefore, it is necessary to examine HER2 expression in other types of solid cancer, such as endometrial cancer, to identify potential candidate tumors for new HER2targeted treatments.

Combination therapy including a VEGFR2 antibody has been highlighted for treatment-resistant cancer. In 2012 , Kodack et al. generated a mouse model of HER2-amplified breast cancer with brain metastasis. Brain metastatic lesions were not affected by treatment with trastuzumab alone; however, combination therapy with lapatinib and an antibody to VEGFR2 slowed tumor growth and progression (20).

There is an urgent need to identify potential biomarkers of an effective response to targeted endometrial cancer therapies. To date, biomarkers are not routinely used in the clinical treatment or diagnosis of endometrial cancer. In this study, we examined the expression of HER2 and VEGFR2 in a tissue microarray (TMA) of tissue from patients with endometrial cancer and potential statistical associations with clinicopathological data. In addition, the relationship between HER2 and VEGFR2 was examined in Ishikawa cells, an endometrial cancer cell line. In particular, potential differences in cell migration and proliferation were evaluated after HER2 knockdown. Herein, we studied the mechanisms of action of HER2 and VEGFR2 in early-stage endometrial carcinoma and elucidated the possibility of their application as prognostic and therapeutic indicators.

\section{Materials and Methods}

Case selection. Specimens from 60 patients who underwent hysterectomy for endometrioid-type endometrial cancer between January 2002 and December 2009 at Gyeongsang National University Hospital, Jinju, Republic of Korea, were selected. Hematoxylin and eosin-stained sections on glass slides were reviewed by two pathologists. Electronic medical records were reviewed, and clinicopathological data, including age, T-stage, and histological grade, were obtained. Cancer stage was determined according to the Seventh Edition of the American Joint Committee on Cancer (21). Tumor histological type and grade were determined as per the Fourth Edition of the World Health Organization classification (22). This study was approved by the Institutional Review Board of Gyeongsang National University Hospital with a waiver of informed consent (GNUH-2019-03-009).

TMA and immunohistochemistry. Representative hematoxylin and eosin-stained sections on glass slides containing prominent intratumoral regions were chosen from among the 60 endometrial cancer specimens. One core was obtained from the invasive front of each representative paraffin block and transferred to a recipient TMA block.

Immunohistochemical staining was performed on the TMA blocks. A monoclonal antibody against HER2 (1:250 dilution, \#ab214275; Abcam, Cambridge, MA, USA) and anti- polyclonal antibody to VEGFR2 (1:500 dilution, \#ab39256; Abcam) were used as the primary antibodies. The pattern of immunohistochemical staining was evaluated; in particular, membranous expression of HER2 and cytoplasmic expression of VEGFR2 were noted. The intensity of stained tumor cells was graded as either high or low. Tumor cells with an intensity higher than that of stromal cells and lymphocytes were graded as high, and those with an intensity the same or lower than that of non-tumor cells were graded as low.

Cell culture and knockdown of HER2 and VEGFR2. The human endometrial cancer cell line Ishikawa was provided by Dr. Myoungseok Han (University of Dong-A). Ishikawa cells were cultured in Dulbecco's modified Eagle's medium (Gibco, Thermo Fisher Scientific, Waltham, MA, USA) supplemented with $10 \%$ fetal bovine serum Gibco and 1\% penicillin-streptomycin (Corning, Glendale, AZ, USA) at $37^{\circ} \mathrm{C}$ in an atmosphere containing $5 \% \mathrm{CO}_{2}$. Ishikawa cells were cultured to $70-80 \%$ confluence in 60-mm dishes. The cells were transfected using Lipofectamine 3000 (Invitrogen, Thermo Fisher Scientific) with human HER2 siRNA (siHER2, \#20642; Bioneer, Oakland, CA , USA), VEGFR2 siRNA (siVEGFR2, \#3791-3; Bioneer) or negative control scrambled siRNA (\#SN-1002; Bioneer) at a final concentration of $50 \mathrm{nM}$. After 24 hours of incubation, the cells were re-transfected using the same protocol described above. The cells were incubated for $72 \mathrm{~h}$ before harvest.

Semiquantitative polymerase chain reaction (PCR). Total RNA was extracted from cells using TRIzol reagent (Qiagen). Total RNA was quantified using a NanoDrop 2000 (Thermo Fisher Scientific), and $1 \mu \mathrm{g}$ of total RNA was reverse transcribed to cDNA using a Maxime RT PreMix Kit (iNtRON, Burlington, MA, USA). Equal amounts of synthesized cDNA were used for semiquantitative PCR using the Maxime PCR PreMix kit (iNtRON, Primers specific for HER2 (\#P128755; Bioneer) and VEGFR2 (\#P308687; Bioneer) were used. The primer sequences used for glyceraldehyde 3-phosphate 
dehydrogenase (GAPDH) were as follows: Forward: 5'-GTC CAC CAC CCT GTT GCT GTA G-3', and reverse: 5'-CAA GGT CAT CCA TGA CAA CTT TG-3'.

Western blot analysis. Proteins were extracted using RIPA lysis buffer (Thermo Fisher Scientific) containing protease inhibitor cocktail (Thermo Fisher Scientific). The total protein concentration of each cell lysate was measured by the Bradford method using bovine serum albumin as a standard. Equal amounts of protein lysate $(45 \mu \mathrm{g})$ were separated on a denaturing polyacrylamide gel and then transferred to a nitrocellulose membrane. The following primary antibodies were used for immunoblotting: anti-HER2 (\#ab214275; Abcam), anti-VEGFR2 (\#ab39256; Abcam), and antiGAPDH (\#ab8245; Abcam). Subsequently, the membranes were incubated with horseradish peroxidase-conjugated secondary antibodies and developed by enhanced chemiluminescence (Thermo Fisher Scientific Digital chemiluminescence images were captured by FUSION Solo (Vilber, Collégien, France).

Wound-healing assays. Ishikawa cells were transfected with siHER2 as described above. Once the cells reached $100 \%$ confluence, 25 Culture-Inserts 2 Well for Self-Insertion (\#80209; Ibidi, Planegg, Germany) were used for the wound-healing assays. The cells were washed twice with phosphate-buffered saline to remove detached cells. Then the cells were incubated at $37^{\circ} \mathrm{C}$ in an atmosphere containing $5 \% \mathrm{CO}_{2}$, and the wounded area was monitored using JuLI Br (NanoEntek, Waltham, MA, USA). Images were transferred to and analyzed by image-processing software ImageJ (National Institutes of Health, Bethesda, MD, USA).

Statistical analysis. Association analyses were performed using the chi-square test and Fisher's exact test. A value of $p<0.05$ was considered to indicate statistical significance. All data were analyzed using SPSS ver. 24.0 (IBM Corp., Armonk, NY, USA).

\section{Results}

Patient characteristics. The clinicopathological features of the 60 patients with endometrioid-type endometrial cancer are summarized in Table I. The mean age was 51 years. All patients underwent hysterectomy. Among the 60 cases, 55 $(91.7 \%)$ were classified as stage I after surgery, whereas 40 $(66.7 \%)$ were considered FIGO histological grade 1.

Images of immunohistochemical staining for HER2 and VEGFR2 are shown in Figure 1 and Figure 2. There were more cases of low than high HER2 expression $(68.3 \%$ vs $31.7 \%$ ) but the percentages of samples with low and high expression of VEGFR2 were similar ( $51.7 \% \mathrm{vs} .48 .3 \%)$.

HER2 and VEGFR2 expression in endometrial cancer. Table II shows the clinicopathological data according to the HER2 and VEGFR2 expression patterns for the 60 patients with endometrioid-type endometrial cancer. In the TMA, the higher the expression of VEGFR2, the lower the FIGO grade. High VEGFR2 expression was observed in FIGO grade 1 samples, and low VEGFR2 expression was observed in samples of FIGO grade 2 or higher (chi-square test, $p=0.044$ ).
Table I. Clinicopathological information of patients $(n=60)$.

\begin{tabular}{llc}
\hline Variable & & Value \\
\hline Age, years & Mean (range) & $51(35-78)$ \\
T-Stage, n (\%) & Ia & $43(71.7)$ \\
& Ib & $12(20)$ \\
& II & $3(5)$ \\
& IIIa & $1(1.7)$ \\
Histological grade, n (\%) & IIIb & $1(1.7)$ \\
& 1 & $40(66.7)$ \\
HER2 expression, n (\%) & 3 & $15(25)$ \\
& Low & $5(8.3)$ \\
VEGFR2 expression, n (\%) & High & $41(68.3)$ \\
& Low & $19(31.7)$ \\
& High & $31(51.7)$ \\
\end{tabular}

HER2: Human epidermal growth factor receptor 2; VEGFR2: vascular endothelial growth factor receptor 2 .

The positive correlation between HER2 and VEGFR2 expression is shown in Table III. The association between HER2 and VEFR2 expression was statistically significant in T1 stage $(p=0.002)$ and FIGO grade 1 tumors $(p=0.004)$. In the case of low expression of both VEGFR2 and HER2, the $\mathrm{T}$ stage was low, and the FIGO grade was also low.

HER2 and VEGFR2 expression in Ishikawa endometrial cancer cells. To extend our immunohistochemical results demonstrating an association between HER2 and VEGFR2, we evaluated the expression of HER2 and VEGFR2 in Ishikawa cells, an endometrial cancer cell line. Both HER2 and VEGFR2 were highly expressed in the Ishikawa cell line. To identify the association between HER2 and VEGFR2, each target was knocked down to observe the change in the other protein, but there were no significant changes. Transfection of Ishikawa cells with HER2 siRNA caused no significant change in the mRNA (Figure 3A) or protein (Figure 3B) levels of VEGFR2 compared with transfection of cells with scrambled control siRNA. Transfection of Ishikawa cells with VEGFR2 siRNA also evoked no significant change in the mRNA (Figure 4A) or protein levels (Figure 4B) of HER2 compared with scrambled control siRNA transfection. In the Ishikawa cell line, no significant association was found between HER 2 and VEGFR2 expression.

HER2 silencing inhibited endometrial cancer cell migration and proliferation. During the 36 hours of the wound-healing assay, there were significant differences between the HER2 knockdown and the control cells (Figure 5A), as measured by ImageJ. HER2 knockdown slowed the migration of endometrial cancer cells. Cell confluence after 60 hours differed markedly between cells with HER2 knockdown and 


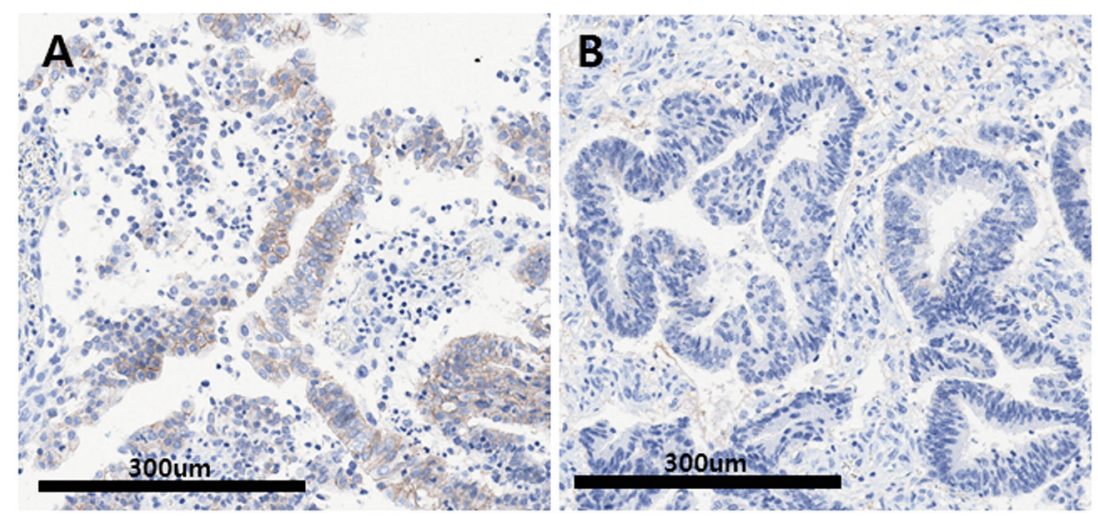

Figure 1. Representative images of human epidermal growth factor receptor 2 (HER2) staining patterns. A: Strong positive and distinct membranous staining of HER2 in endometrial cancer (x200). B: Negative expression of HER2 (×200).
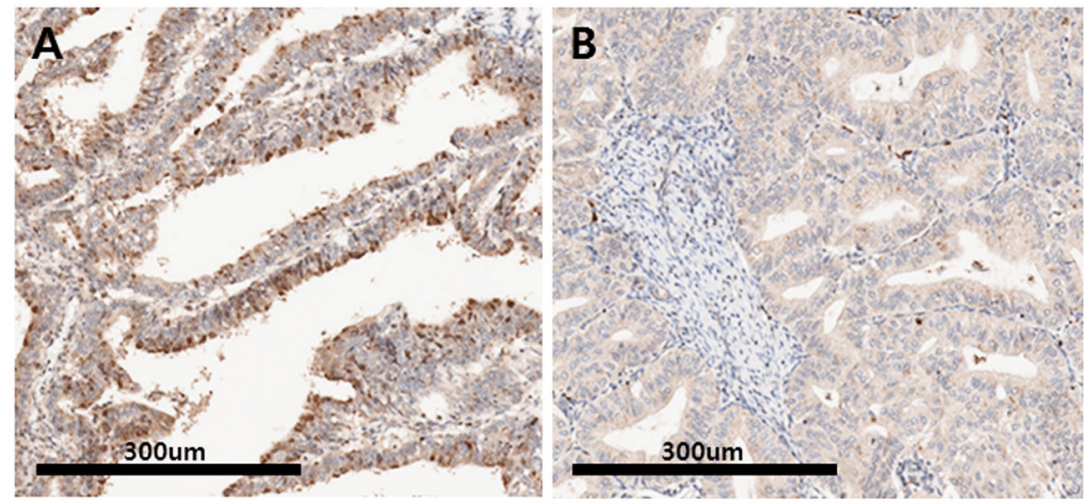

Figure 2. Representative images of vascular endothelial growth factor receptor 2 (VEGFR2) staining patterns. A: Strong positive and distinct cytoplasmic staining of VEGFR2 in endometrial cancer $(\times 200)$. B: Mild expression of VEGFR2 $(\times 200)$.

control cells at $70 \%$ vs. 87\%, respectively (Figure 5B), demonstrating that cell proliferation involves HER2 activity.

\section{Discussion}

Precise risk stratification is required when diagnosing endometrial cancer to implement the appropriate treatment strategy. In this study, we examined the association of stage, histopathological features and biomarker levels in endometrial cancer. We identified the relationship between VEGFR2 and HER2 expression in low FIGO-grade and low T-stage cancer and demonstrated the tendency of tumor cell migration to decrease when HER2 expression is low.

Endometrial cancer is diagnosed by endometrial biopsy or curettage. The histological information obtained at diagnosis has the potential to help in selecting the therapeutic strategy (23). The standard treatment for endometrial cancer is primary hysterectomy and bilateral salpingo-oophorectomy, with lymph node surgery and adjuvant treatment being added according to the histology and stage (24). The FIGO (25) and TNM classification (22) are the most utilized staging classifications.

Our immunohistochemical studies showed that expression of VEGFR2 was inversely associated with FIGO grade. This means that FIGO grade can be estimated if VEGFR2 staining is performed on a sample obtained at the time of endometrial cancer diagnosis, although this grade can only be confirmed by surgery; thus, a more specific treatment plan can be established. Kotowitz et al. reported that in patients with endometrial cancer, serum VEGF values were significantly higher in those with para-aortic lymph node metastases than in those without such lesion (17). Abbink et al. showed that in patients with endometrial cancer, elevated serum levels of VEGF were associated with poor prognosis and known clinicopathological factors of recurrent disease (16). Oplawski et al. reported that the higher the grade of endometrial cancer, the higher the protein expression level of VEGFD and VEGFR3 protein through tissue immunostaining (15). 
Table II. Clinicopathological data for 60 uterine endometrioid carcinomas according to human epidermal growth factor receptor 2 (HER2) and vascular endothelial growth factor receptor 2 (VEGFR2) expression.

\begin{tabular}{|c|c|c|c|c|c|c|c|}
\hline & & \multicolumn{3}{|c|}{ HER2, n (\%) } & \multicolumn{3}{|c|}{ VEGFR2, n (\%) } \\
\hline & & Low & High & $p$-Value & Low & High & $p$-Value \\
\hline \multirow[t]{2}{*}{ Age* } & $\leq 50$ Years & $20(33.3)$ & $6(10)$ & 0.211 & $9(15)$ & $17(28.3)$ & 0.021 \\
\hline & $\geq 51$ Years & $21(35)$ & $13(21.7)$ & & $22(36.7)$ & $12(20)$ & \\
\hline \multirow[t]{2}{*}{ T-Stage } & 1 & $39(65)$ & $16(26.7)$ & 0.314 & $28(46.7)$ & $27(45)$ & $>0.99$ \\
\hline & $\geq 2$ & $2(3.3)$ & $3(5)$ & & $3(5)$ & $2(3.3)$ & \\
\hline \multirow[t]{2}{*}{ FIGO grade } & 1 & $28(46.7)$ & $12(20)$ & 0.695 & $17(28.3)$ & $23(38.4)$ & 0.044 \\
\hline & $\geq 2$ & $13(21.7)$ & 7 (11.6) & & $14(23.3)$ & $6(10)$ & \\
\hline
\end{tabular}

FIGO: International Federation of Obstetrics and Gynecology classification. *Median=51 years.

Table III. Association between (HER2) and vascular endothelial growth factor receptor 2 (VEGFR2) expression.

\begin{tabular}{|c|c|c|c|c|c|c|c|c|c|c|c|c|}
\hline & \multicolumn{6}{|c|}{ T-Stage } & \multicolumn{6}{|c|}{ FIGO grade } \\
\hline & \multicolumn{3}{|c|}{ Low (T1) } & \multicolumn{3}{|c|}{ High $(\geq \mathrm{T} 2)$} & \multicolumn{3}{|c|}{ Low (1) } & \multicolumn{3}{|c|}{$\operatorname{High}(\geq 2)$} \\
\hline & \multicolumn{12}{|c|}{ HER2 } \\
\hline VEGFR2 & Low & High & $p$-Value & Low & High & $p$-Value & Low & High & $p$-Value & Low & High & $p$-Value \\
\hline Low, n (\%) & 25 (41.7) & $3(5)$ & 0.002 & $1(1.7)$ & $2(3.3)$ & $>0.99$ & $16(26.7)$ & $1(1.7)$ & 0.004 & $10(16.7)$ & $4(6.7)$ & 0.613 \\
\hline High, n (\%) & $14(23.3)$ & 13 (21.7) & & $1(1.7)$ & $1(1.7)$ & & $12(20)$ & $11(18.3)$ & & $3(5)$ & $3(5)$ & \\
\hline
\end{tabular}

In the present study, there was a statistically significant inverse association between HER2 expression and VEGFR2 expression in T1 stage and FIGO grade 1 cancer. When levels of both VEGFR2 and HER2 were low, the T-stage and FIGO grade were also low. To our knowledge, this is the first report on the relationship between VEGFR2 and HER2 in endometrial cancer.

The endometrial cancer cell experiments showed a different trend from the TMA results. Both HER2 and VEGFR2 were highly expressed in the Ishikawa endometrial cancer cell line, but no significant correlation between HER2 and VEGFR2 was found. The main reason for this result may be that unlike TMAs, it is difficult to take into consideration the tumor microenvironment in cell experiments (26). In cell experiments, only tumor cells are involved, but other factors are involved in human tissue, as captured in TMAs, hence the results may be different.

Wound-healing assays are commonly used to study cell migration and the underlying biology (27). Our data demonstrated that in HER2 knockdown cells, cell migration occurred more slowly than in the control cells, and the cell confluence observed after 60 hours was also lower than that for the control. Knockdown of HER2 in Ishikawa cells reduced cell migration and proliferation. HER2-expressing breast cancer has a higher histological grade and a more aggressive nature (19). Mori et al. reported that HER2 knockdown affected pathways involved in endometrial carcinogenesis and increased sensitivity to paclitaxel (28). Regarding the efficacy of anti-HER2 therapy in endometrial cancer, clear results have not yet been reported due to incompatibility or heterogeneity among study subjects (29, 30). However, our results support the possible application of anti-HER2 therapy in endometrial cancer.

This study had some limitations. Only 60 patients were involved, and most of the cases were of early-stage endometrial cancer. Therefore, a larger study sample size is needed to validate the mechanisms regulating the expression of HER2 and VEGFR2 in early and advanced endometrial cancer.

The mechanism of HER2 and VEGFR2 expression in early endometrial cancer may differ from that in advanced cancer. Based on this hypothesis, a previous study reported that the adenoma-carcinoma sequence in colon cancer, which underlies the progression from precancerous lesions to invasive cancer, is governed by the accumulation of mutations (31). Examining the mechanisms controlling HER2 and VEGFR2 expression in early endometrial cancer, i.e., that confined to the endometrium, may contribute to the elucidation of the tumorigenesis of endometrial cancer.

In conclusion, our study revealed the association of VEGFR2 and HER2 expression in cancer of low FIGO grade 
A
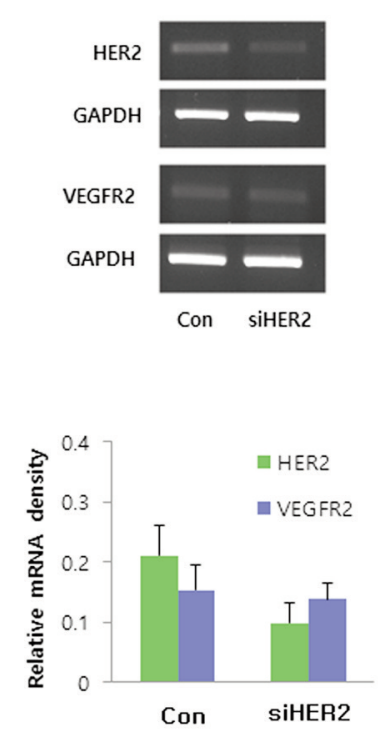

B CON HER2 SIRNA
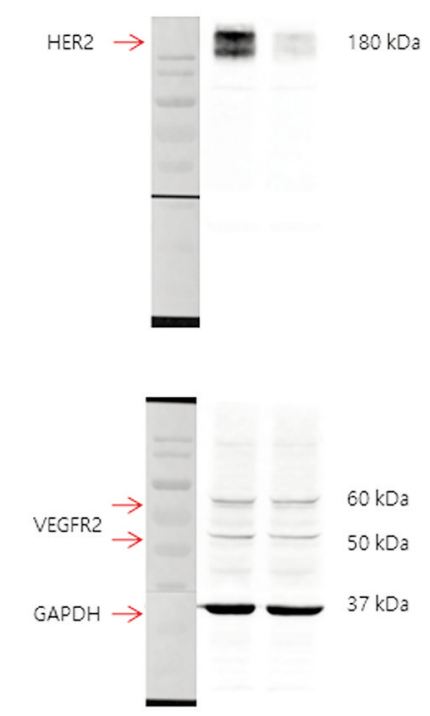

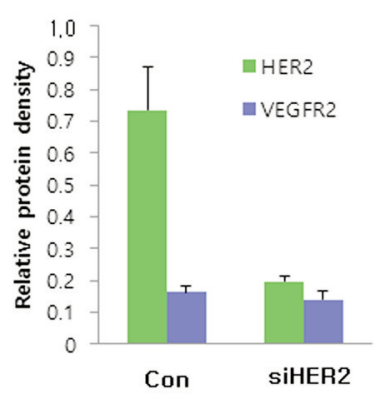

Figure 3. The mRNA (A) and protein (B) expression levels of human epidermal growth factor receptor 2 (HER2) and vascular endothelial growth factor receptor 2 (VEGFR2) in Ishikawa cells transfected with HER2 siRNA. A: Transfection of Ishikawa cells with HER2 siRNA did not significantly affect VEGFR2 mRNA levels B: Transfection of Ishikawa cells with HER2 siRNA did not significantly affect VEGFR2 protein levels. Data are the mean \pm standard deviation $(S D)$ of three independent experiments.

A
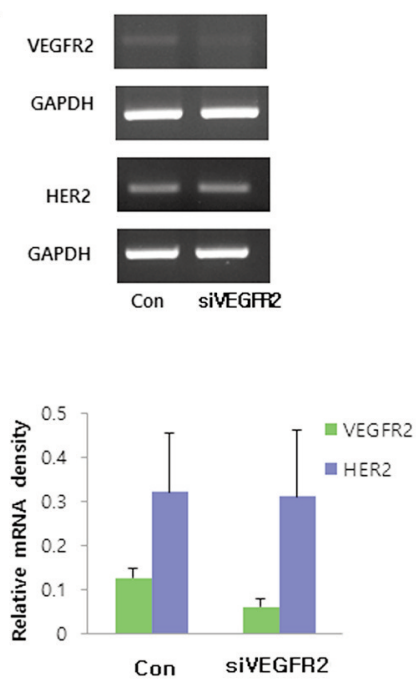

B
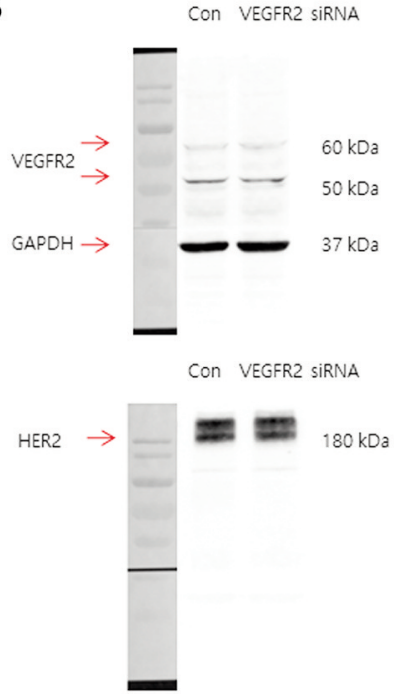

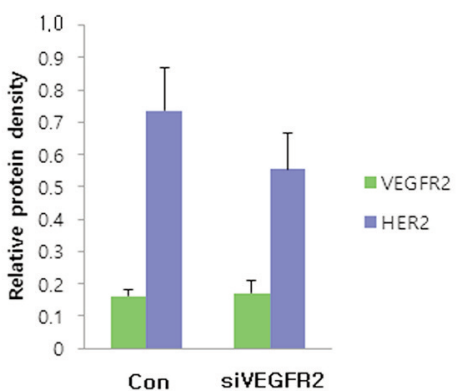

Con siVEGFR2

Figure 4. The mRNA (A) and protein (B) expression levels of human epidermal growth factor receptor 2 (HER2) and vascular endothelial growth factor receptor 2 (VEGFR2) in Ishikawa cells transfected with VEGFR2 siRNA. A: Transfection of Ishikawa cells with VEGFR2 siRNA did not significantly affect HER2 mRNA levels B: Transfection of Ishikawa cells with VEGFR2 siRNA did not significantly affect HER2 protein levels. Data are the mean \pm standard deviation (SD) of three independent experiments.

and T-stage. Since VEGFR2 expression was also identified as being correlated with FIGO histological grade here, this may help clinicians plan treatment strategy by predicting the cancer stage and prognosis before surgery based on samples obtained from diagnostic curettage. In addition, tumor cell migration and proliferation tended to decrease when HER2 expression was reduced in Ishikawa cells. This finding suggests the possibility of using anti-HER2 therapy for endometrial cancer. 
A
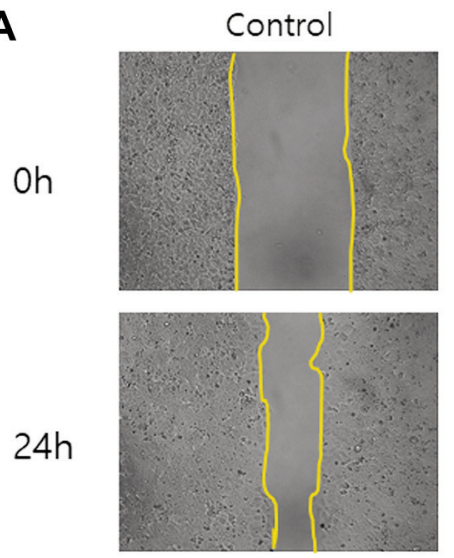

$30 \mathrm{~h}$

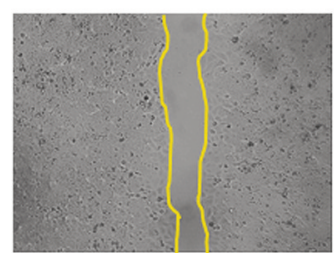

$36 \mathrm{~h}$

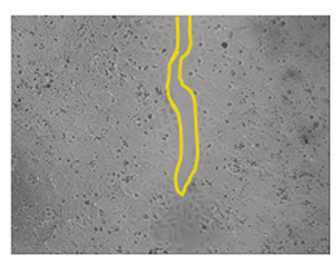

SIHER2
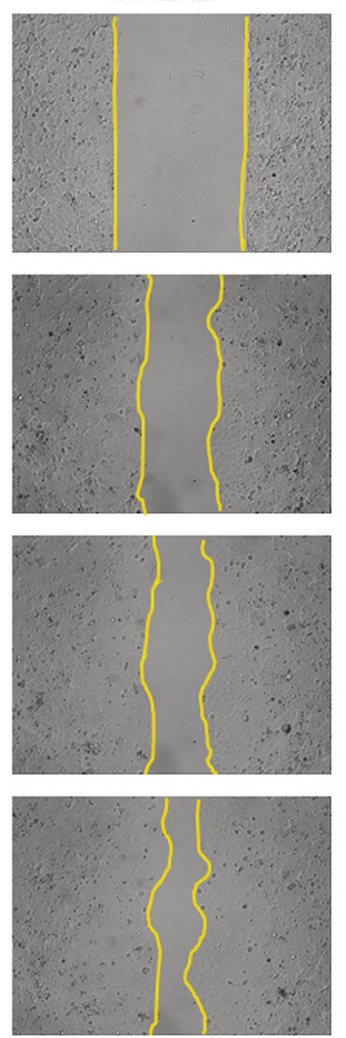

B

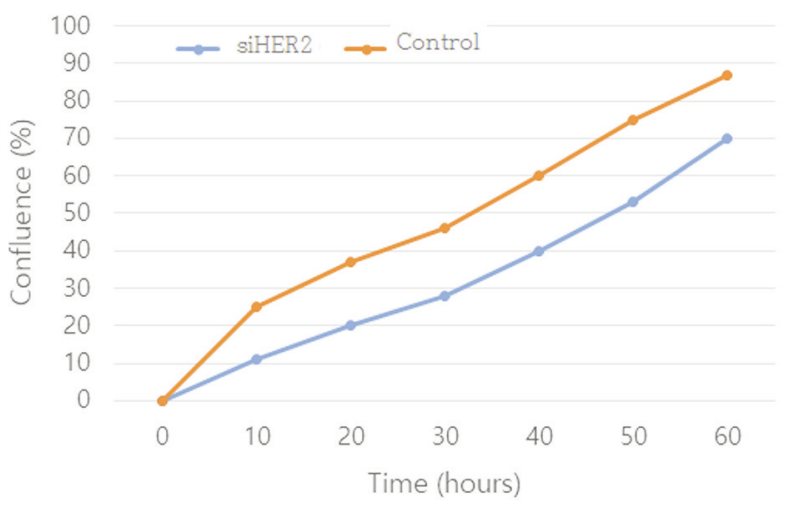

Figure 5. Effects of human epidermal growth factor receptor 2 (HER2) knockdown on Ishikawa cell migration and proliferation. A: During the 36 hours of the wound-healing assay, the migration of endometrial cancer cells was slowed in HER2 knockdown cells compared to the control. B: Cell confluence after 60 hours differed markedly between cells with HER2 knockdown and control cells, at 70\% vs. 87\%, respectively.

\section{Conflicts of Interest}

The Authors report no conflicts of interest.

\section{Authors' Contributions}

HJ An, DH Song and JE Park conceived and designed the experiments. HJ An, DH Song, YM Kim, HC Jo, JC Baek and JE Park performed the experiments. HJ An, DH Song and JE Park analyzed the data. HJ An and JE Park wrote the article. DH Song supervised the experiments and reviewed the article.

\section{Acknowledgements}

This work was supported by a National Research Foundation of Korea (NRF) grant funded by the Korean government (MSIT) (No. 2021R1G1A1094975).

\section{References}

1 Sung H, Ferlay J, Siegel RL, Laversanne M, Soerjomataram I, Jemal A and Bray F: Global cancer statistics 2020: GLOBOCAN estimates of incidence and mortality worldwide for 36 cancers in 185 countries. CA Cancer J Clin 71(3): 209-249, 2021. PMID: 33538338. DOI: $10.3322 /$ caac. 21660

2 Ha HI, Chang HK, Park SJ, Lim J, Won YJ and Lim MC: The incidence and survival of cervical, ovarian, and endometrial cancer in Korea, 1999-2017: Korea Central Cancer Registry. Obstet Gynecol Sci 64(5): 444-453, 2021. PMID: 34399564. DOI: $10.5468 /$ ogs.21116

3 Urick ME and Bell DW: Clinical actionability of molecular targets in endometrial cancer. Nat Rev Cancer 19(9): 510-521, 2019. PMID: 31388127. DOI: 10.1038/s41568-019-0177-x

4 Kong A, Johnson N, Kitchener HC and Lawrie TA: Adjuvant radiotherapy for stage I endometrial cancer: an updated Cochrane systematic review and meta-analysis. J Natl Cancer Inst 104(21): 1625-1634, 2012. PMID: 22962693. DOI: 10.1093/jnci/djs374

5 Obel JC, Friberg G and Fleming GF: Chemotherapy in endometrial cancer. Clin Adv Hematol Oncol 4(6): 459-468, 2006. PMID: 16981669.

6 Gaber-Wagener A and Marth C: Role of hormonal therapy in advanced stage endometrial cancer. In: Management of Endometrial Cancer. Mirza MR (ed.) Springer, pp. 243-248, 2020.

7 Rosen LS: Angiogenesis inhibition in solid tumors. Cancer J 7(Suppl 3): S120-S128, 2001. PMID: 11779082. 
8 Folkman J: Angiogenesis inhibitors: a new class of drugs. Cancer Biol Ther 2(4 Suppl 1): S127-S133, 2003. PMID: 14508090.

9 Nicholson B and Theodorescu D: Angiogenesis and prostate cancer tumor growth. J Cell Biochem 91(1): 125-150, 2004. PMID: 14689586 . DOI: $10.1002 /$ jcb.10772

10 Allen J and Bergsland EK: Angiogenesis in colorectal cancer: therapeutic implications and future directions. Hematol Oncol Clin North Am 18(5): 1087-119, ix, 2004. PMID: 15474337. DOI: 10.1016/j.hoc.2004.05.002

11 Bevacizumab (Avastin). Med Lett Drugs Ther 46(1184): 47-48, 2004. PMID: 15212050.

12 Zondor SD and Medina PJ: Bevacizumab: an angiogenesis inhibitor with efficacy in colorectal and other malignancies. Ann Pharmacother 38(7-8): 1258-1264, 2004. PMID: 15187215. DOI: $10.1345 / \mathrm{aph} .1 \mathrm{D} 470$

13 Hurwitz H, Fehrenbacher L, Novotny W, Cartwright T, Hainsworth J, Heim W, Berlin J, Baron A, Griffing S, Holmgren E, Ferrara N, Fyfe G, Rogers B, Ross R and Kabbinavar F: Bevacizumab plus irinotecan, fluorouracil, and leucovorin for metastatic colorectal cancer. N Engl J Med 350(23): 2335-2342, 2004. PMID: 15175435. DOI: 10.1056/NEJMoa032691

14 Ferrara N, Hillan KJ, Gerber HP and Novotny W: Discovery and development of bevacizumab, an anti-VEGF antibody for treating cancer. Nat Rev Drug Discov 3(5): 391-400, 2004. PMID: 15136787 . DOI: $10.1038 / \mathrm{nrd} 1381$

15 Oplawski M, Dziobek K, Zmarzły N, Grabarek B, Halski T, Januszyk P, Kuś-Kierach A, Adwent I, Dąbruś D, Kiełbasiński $\mathrm{K}$ and Boron D: Expression profile of VEGF-C, VEGF-D, and VEGFR-3 in different grades of endometrial cancer. Curr Pharm Biotechnol 20(12): 1004-1010, 2019. PMID: 31333122. DOI: $10.2174 / 1389201020666190718164431$

16 Abbink K, Zusterzeel PLM, Geurts-Moespot A, van der Steen R, Span PN and Sweep FCGJ: Prognostic significance of VEGF and components of the plasminogen activator system in endometrial cancer. J Cancer Res Clin Oncol 146(7): 1725-1735, 2020. PMID: 32394054. DOI: 10.1007/s00432-020-03225-7

17 Kotowicz B, Fuksiewicz M, Jonska-Gmyrek J, Berezowska A, Radziszewski J, Bidzinski $\mathrm{M}$ and Kowalska M: Clinical significance of pretreatment serum levels of VEGF and its receptors, IL- 8, and their prognostic value in type I and II endometrial cancer patients. PLoS One 12(10): e0184576, 2017. PMID: 28991928. DOI: 10.1371/journal.pone.0184576

18 Oh DY and Bang YJ: HER2-targeted therapies - a role beyond breast cancer. Nat Rev Clin Oncol 17(1): 33-48, 2020. PMID: 31548601. DOI: 10.1038/s41571-019-0268-3

19 Loibl S and Gianni L: HER2-positive breast cancer. Lancet 389(10087): 2415-2429, 2017. PMID: 27939064. DOI: 10.1016/ S0140-6736(16)32417-5

20 Kodack DP, Chung E, Yamashita H, Incio J, Duyverman AM, Song Y, Farrar CT, Huang Y, Ager E, Kamoun W, Goel S, Snuderl M, Lussiez A, Hiddingh L, Mahmood S, Tannous BA, Eichler AF, Fukumura D, Engelman JA and Jain RK: Combined targeting of HER2 and VEGFR2 for effective treatment of HER2-amplified breast cancer brain metastases. Proc Natl Acad Sci USA 109(45): E3119-E3127, 2012. PMID: 23071298. DOI: 10.1073/pnas.1216078109
21 Edge SB and Compton CC: The American Joint Committee on Cancer: the 7th edition of the AJCC cancer staging manual and the future of TNM. Ann Surg Oncol 17(6): 1471-1474, 2010. PMID: 20180029. DOI: 10.1245/s10434-010-0985-4

22 Kurman RJ, Carcangiu ML, Herrington CS and Young RH (eds.): WHO Classification of Tumours of Female Reproductive Organs. Geneva, IARC, 2014.

23 Touboul C, Piel B, Koskas M, Gonthier C, Ballester M, Cortez $\mathrm{A}$ and Daraï E: Factors predictive of endometrial carcinoma in patients with atypical endometrial hyperplasia on preoperative histology. Anticancer Res 34(10): 5671-5676, 2014. PMID: 25275072.

24 Morice P, Leary A, Creutzberg C, Abu-Rustum N and Darai E: Endometrial cancer. Lancet 387(10023): 1094-1108, 2016. PMID: 26354523. DOI: 10.1016/S0140-6736(15)00130-0

25 Creasman W: Revised FIGO staging for carcinoma of the endometrium. Int J Gynaecol Obstet 105(2): 109, 2009. PMID: 19345353. DOI: 10.1016/j.ijgo.2009.02.010

26 Felix AS, Weissfeld J, Edwards R and Linkov F: Future directions in the field of endometrial cancer research: the need to investigate the tumor microenvironment. Eur J Gynaecol Oncol 31(2): 139-144, 2010. PMID: 20527227.

27 Lampugnani MG: Cell migration into a wounded area in vitro. Methods Mol Biol 96: 177-182, 1999. PMID: 10098136. DOI: 10.1385/1-59259-258-9:177

28 Mori N, Kyo S, Nakamura M, Hashimoto M, Maida Y, Mizumoto Y, Takakura M, Ohno S, Kiyono T and Inoue M: Expression of HER-2 affects patient survival and paclitaxel sensitivity in endometrial cancer. Br J Cancer 103(6): 889-898, 2010. PMID: 20664599. DOI: 10.1038/sj.bjc.6605805

29 Fleming GF, Sill MW, Darcy KM, McMeekin DS, Thigpen JT, Adler LM, Berek JS, Chapman JA, DiSilvestro PA, Horowitz IR and Fiorica JV: Phase II trial of trastuzumab in women with advanced or recurrent, HER2-positive endometrial carcinoma: a Gynecologic Oncology Group study. Gynecol Oncol 116(1): 1520, 2010. PMID: 19840887. DOI: 10.1016/j.ygyno.2009.09.025

30 Menderes G, Lopez S, Han C, Altwerger G, Gysler S, Varughese J, Schwartz PE and Santin AD: Mechanisms of resistance to HER2-targeted therapies in HER2-amplified uterine serous carcinoma, and strategies to overcome it. Discov Med 26(141): 39-50, 2018. PMID: 30265854.

31 Kumar V, Abbas A and Aster J: Robbins \& Cotran Pathologic Basis of Disease. Tenth Edition. Elsevier, pp. 814-815, 2020. 